\title{
Research Paper \\ The Effect of Eccentric Retraining on Sensation of Muscle Pain and Delay Onset Muscle Soreness Markers on Sedentary Young Men
}

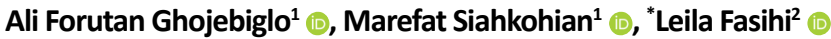

1. Department of Exercise Physiology, Faculty of Educational Sciences and Psychology, University of Mohaghegh Ardabili, Ardabil, Iran 2. Department of Exercise Physiology, Faculty of Physical Education and Sport Sciences, Allameh Tabataba'i University, Tehran, Iran.

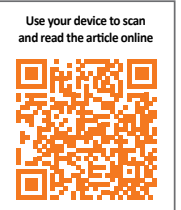

Citation Forutan Ghojebiglo A, Siahkohian M, Fasihi L. The Effect of Eccentric Retraining on Sensation of Muscle Pain and Delay Onset Muscle Soreness Markers on Sedentary Young Men. Scientific Journal of Rehabilitation Medicine. 2021; 10(3):412-423. https://doi. org/10.32598/sjrm.10.3.3

doi $h$ htps://doi.org/10.32598/sjrm.10.3.3

Received: 25 May 2021

Accepted: 06 Jun 2021

Available Online: $23 \mathrm{Jul} 2021$

Keywords:

Muscle pain, Muscle soreness, Exercise,

Creatine kinase, Lactate dehydrogenase

\section{ABSTRACT}

Background and Aims Due to the concerns among trainers about relieving muscle pain as soon as possible, this study aimed to investigate the effect of eccentric retraining on muscle pain and Delay Onset Muscle Soreness (DOMS) markers in sedentary young men.

Methods In this study, sixteen inactive healthy young men voluntarily and after written informed consent. Subjects were randomly divided into control $(n=8)$ and experimental $(n=8)$ groups. Both groups performed seventy eccentric contractions in the flexor elbow muscles with $80 \%$ of maximal repetition. Muscular pain perception, serum levels of Creatine Kinase (CK), and Lactate Dehydrogenase (LDH) indices were measured before, one, 26, 48 and 72 hours after eccentric training. Independent t-test and Repeated measure analysis variance in the significant level of $\mathrm{P} \leq 0.05$ used for data analysis.

Results The sensation of muscle pain and creatine kinase in both groups increased significantly after exercise $(P \leq 0.05)$, lactate dehydrogenase level was increased in both groups. Still, this increase was not significant ( $P>0.05)$. Also, no significant difference in the CK and LDH and pain perception in the measurement stage between the two groups was observed $(P>0.05)$.

Conclusion This study results showed that retraining isn't an essential influential variable on muscle pain and DOMS markers. However, more research needs to be done in this area.

\section{Extended Abstract}

\section{Introduction}

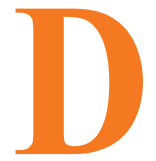

elayed Onset Muscle Soreness (DOMS) is a natural physiological response to unusual physical activity or increased exercise intensity, especially in eccentric resistance activities, which manifests itself in pain, swelling, and decreased range of motion, decreased muscle strength, and discomfort. DOMS appears during the first 24 hours after exercise and peaks between 24 and 72 hours, and takes five to seven days to disappear. Feelings of pain and discomfort associated with muscle soreness can impair athletic performance in training and competitions.

Treatment and prevention of it have become one of the biggest concerns of coaches, practitioners, and sports physiotherapists, so researchers in different ways (Massage, ice massage, vibration, and stimulation of the subcutaneous nerve using exercise) tried to relieve the pain and discomfort associated with the soreness as quickly as possible. But

\section{"Corresponding Author:}

Leila Fasihi, MSc.

Address: Department of Exercise Physiology, Faculty of Physical Education and Sport Sciences, Allameh Tabataba'i University, Tehran, Iran.

Tel: +98 (918) 2183604

E-Mail: I_fasihi@atu.ac.ir 
because the main cause and mechanism of this phenomenon are not yet known precisely, the results of applying these methods can be different.

"Re-training" has been studied as a treatment for the effects of muscle soreness, and researchers have recently reported that re-training significantly reduces the incidence of symptoms or indicators of muscle damage, a phenomenon known as the "repetitive phase effect", Which refers to the effect of muscle adaptation to eccentric exercise. Re-training has been reported to be associated with less destruction of muscle myofibrils, decreased creatine kinase and lactate dehydrogenase enzymes in the blood, and less muscle swelling. Also, the general advice regarding re-training in the period of muscle injury is to avoid stimulating the injured muscle and activity at this time. However, most previous studies have shown the effect of less intense re-training or re-training other than the initial training (eccentric training with concentric re-training) or re-training of two types of muscle contraction simultaneously (simultaneously concentric and eccentric).

Only a few studies have examined the effect of eccentric re-training (exactly the same training and re-training). This limited number did not specify which type of muscle contraction is better for extrinsic exercise-induced muscle injury in practical situations. Still, The effect of eccentric re-training on the healing process of trained muscles is ambiguous. It needs further investigation and research, so this study aimed to investigate the impact of enzyme retraining 24 hours after initial training on muscle soreness and serum levels of Creatine Kinase (CK) and Lactate Dehydrogenase (LDH) enzymes.

\section{Methods}

The present study was quasi-experimental. The statistical population of this study consisted of all inactive healthy students of Mohaghegh Ardabili University with ages ranging from 19-24 years who have not participated in any regular sports activities in the last 2 years. Finally, 16 eligible individuals formed the statistical sample of this study and were randomly divided into two groups: control (eccentric training, $\mathrm{n}=8$ ) and experimental group (eccentric training and re-training, $n=8$ ). At first, during a face-to-face meeting, they were provided with comprehensive information about the research objectives, blood sampling steps, possible risks, and pain after the training program. Then they completed the consent form to participate in the research.

The training session for the experimental group was as follows: first, they warmed-up 10 minutes (including five minutes of walking and jogging and five minutes of soft and stretching movements of the hand and shoulder girdle). Then, to perform eccentric contractions, the subjects of both groups placed their non-superior hand on the table, respectively, and performed 70 eccentric contractions in seven periods of 10 repetitions. Each contraction was $80 \%$ of the maximum repetition of each subject. The subjects of the eccentric re-training group performed the same protocol again after 24 hours. It should be noted that the control group did not participate in any sports activities during the study. Blood samples were taken to measure CK and LDH enzymes at baseline and then at intervals of one, 26, 48, and 72 hours after the protocol. The Visual Analog Scale (VAS) was used to measure the sensation of muscle pain.

On this scale, a $10 \mathrm{~cm}$ graduated horizontal line was drawn on a piece of paper with no pain at the beginning $($ zero $\mathrm{cm})$ and the most severe pain at the end $(10 \mathrm{~cm})$. The subject was then asked with the help of a muscle researcher. Gently stretch the target, indicating the amount of muscle pain felt by a number that best describes it. Shapiro-Wilk statistical test was used to check the normality of data distribution. After confirming the normality of the data, the statistical test of analysis of variance in repeated measures was used to examine the differences between different sampling stages. If significant, the Bonferroni post hoc test was used to determine the differences between different sampling stages. An independent t-test was also used to compare the results of the two groups at each time stage. All calculations were performed using SPSS v. 21 software, at a significance level of $\mathrm{P} \leq 0.05$.

\section{Results}

The independent t-test showed no significant difference between the control and experimental groups regarding changes in muscle pain and $\mathrm{CK}$ at intervals of one, 26, 48 , and 72 hours after activity $(\mathrm{P}>0.05)$. Also, the results of intragroup effects using the Bonferroni post hoc test in the experimental group showed no significant differences in changes in muscle pain sensation, serum CK and LDH levels between the two groups at different post-workout measurements $(\mathrm{P}>0.05)$.

\section{Discussion and Conclusion}

The results of this study showed that performing extroverted retraining did not have a significant effect on the perception of muscle pain and levels of biochemical markers of creatine kinase and lactate dehydrogenase and did not lead to adaptation, so it can be suggested that an eccentric re-training phase 24 hours after Initial exercise can't be an essential variable affecting the amount of pain and indicators of DOMS. However, more research needs to be done. 


\section{Ethical Considerations}

Compliance with ethical guidelines

All ethical principles are considered in this article.

Funding

This study was extracted from the MSc. thesis of the first author at the Department of Exercise Physiology of Mohaghegh Ardabili University.

Authors' contributions

Authors contributed equally in preparing this article.

Conflict of interest

The authors declared no conflict of interest.

Acknowledgments

The authors thank Mohaghegh Ardabili University and the subjects who volunteered to participate in the study. 
تأثير تمرين مجددبرون إ بر احساس درد و شاخصهاى كوفتّى عضلانى تأخيرى در مردان جوان غير فعال

\section{على فروتن قوجه بيكلو' ه، معرفت سياهوهيان' (ـ. "ليلا فصيحى' (1)}

ا. كروه فيزيولوزي ورزشى، دانشكده علوم تربيتى و روانشناسى، دانشكاه محقق اردبيلى، اردبيل، ايران.

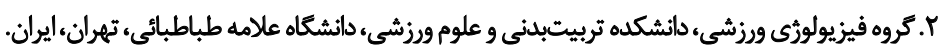

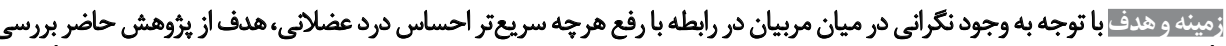

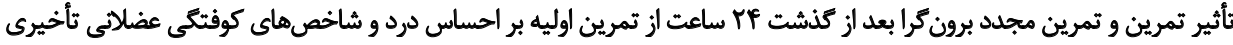

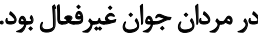

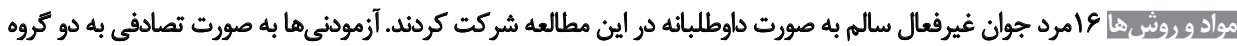

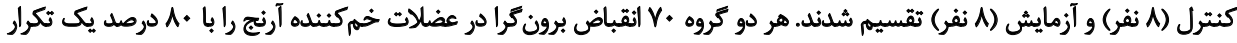

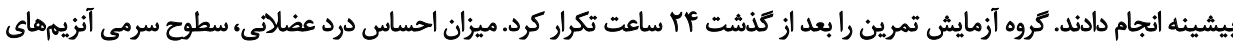

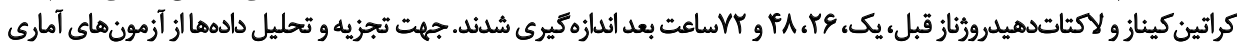

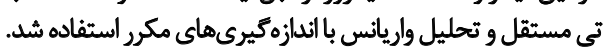

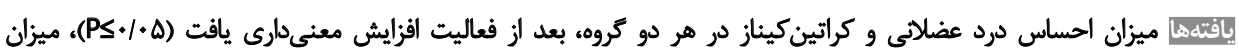

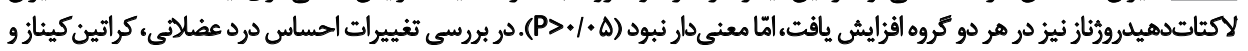

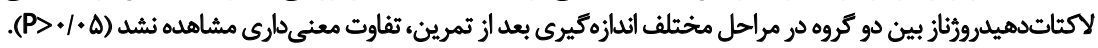

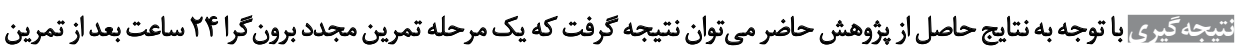

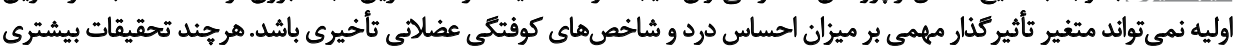

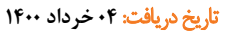

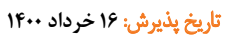

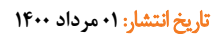

دوره بركشت به حالت اوليه است. درواقع جهت ايجاد توازن

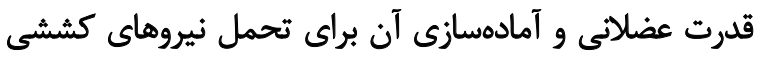

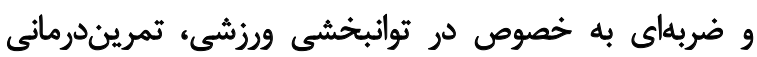

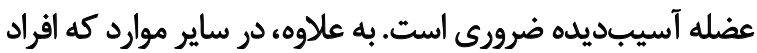

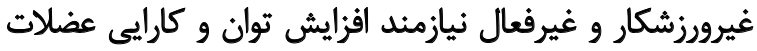

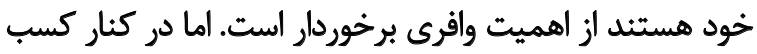

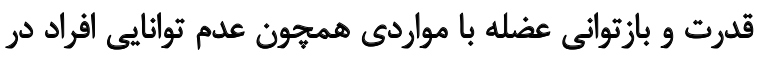

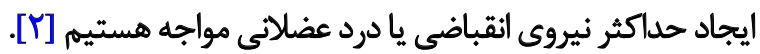

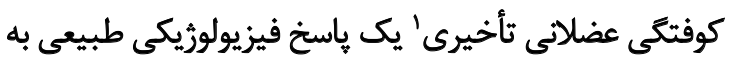

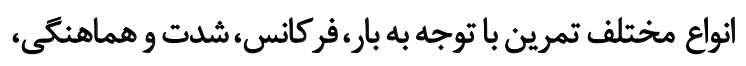

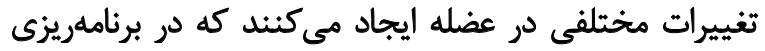

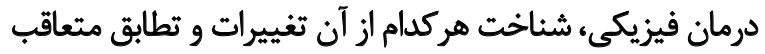

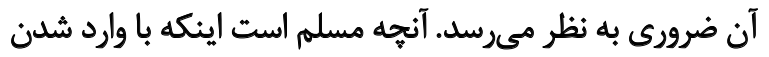

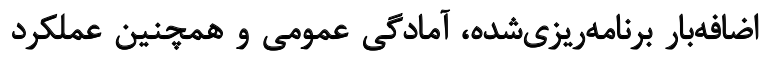

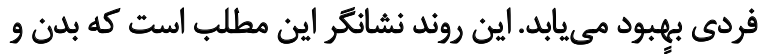

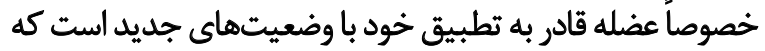

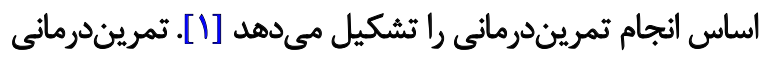

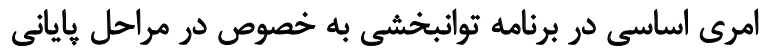




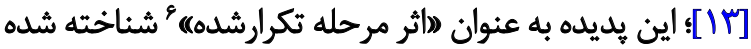

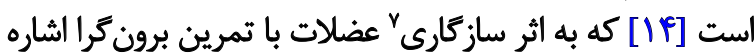

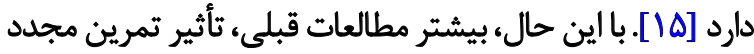

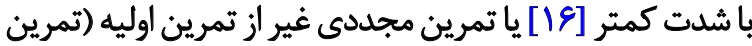

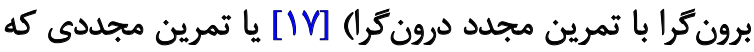

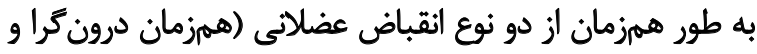

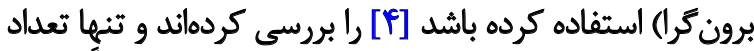

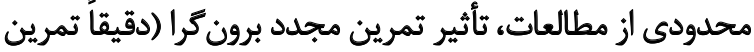

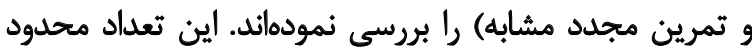

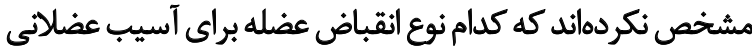

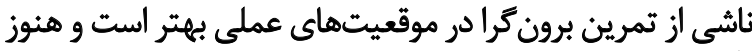

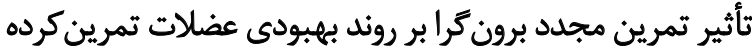

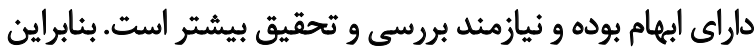

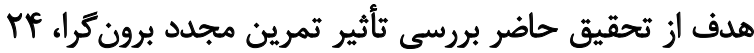

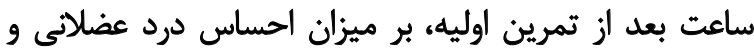

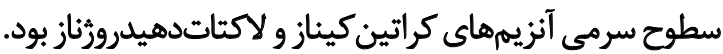

\section{مواد و روشها}

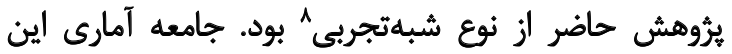

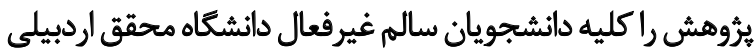

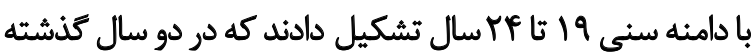

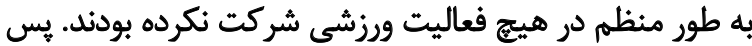

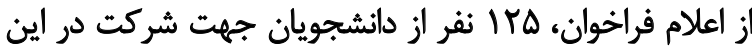

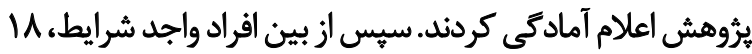

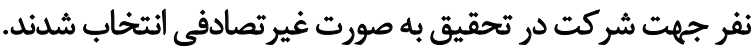

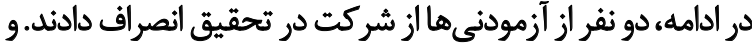

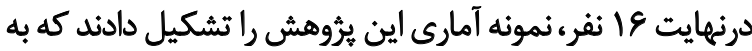

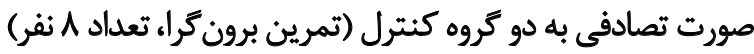

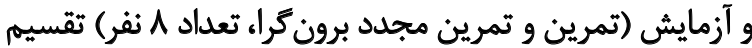

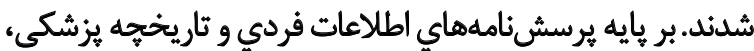

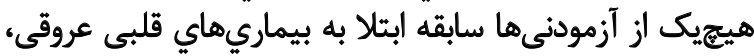

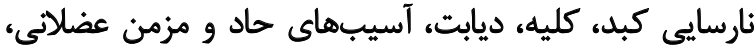

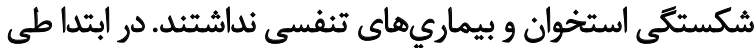

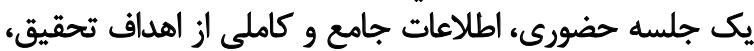

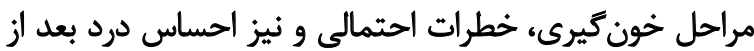

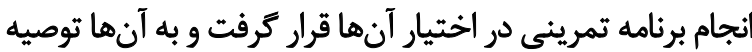

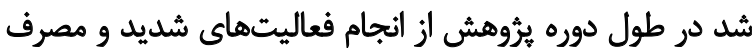

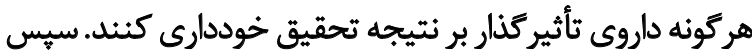

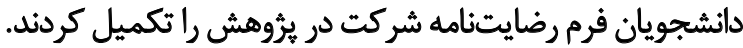

\section{Repeated bout effect}

7. Adaptation effect

8. Quasi-experimental
فعاليت بدنى غيرمعمول، يا افزايش شدت تمرين به خصوص در بر برو

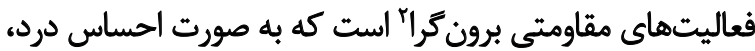

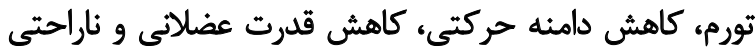

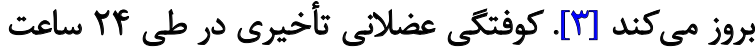

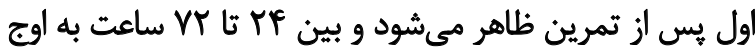

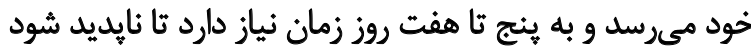

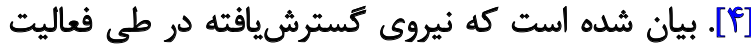
برون

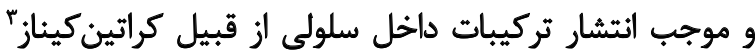

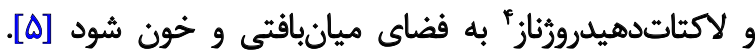

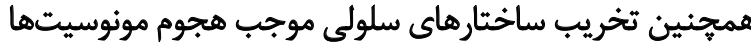

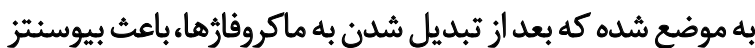

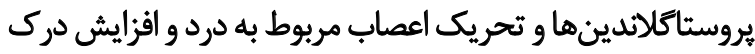

كوفتكى عضله مي شود [9].

احساس درد و ناراحتى همراه با كوفتكى عضلاتى مي تواند

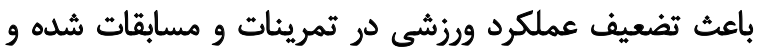

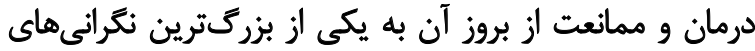

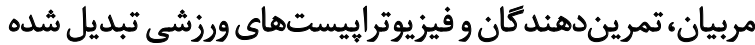

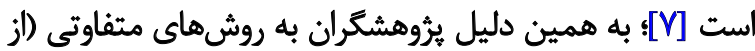

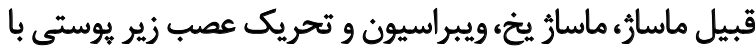

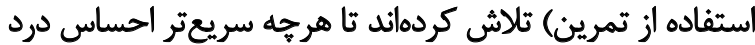

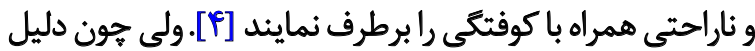

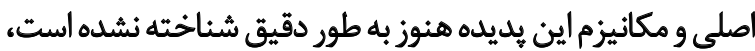

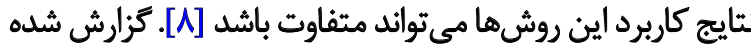

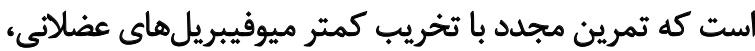

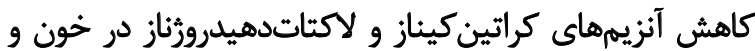

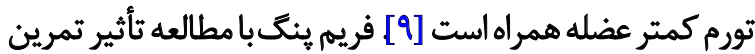

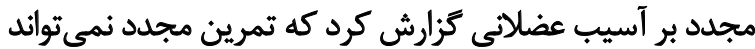

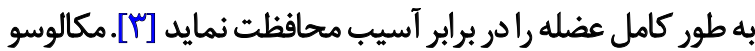

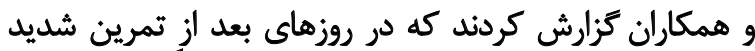

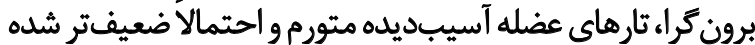

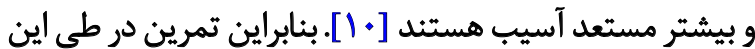

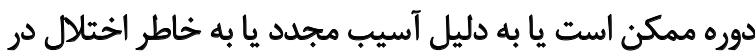

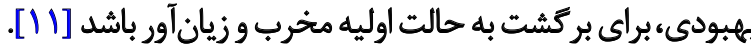

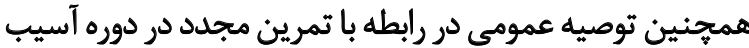
عضلانى اين است كه در اين زمان از تحرين إنريك عضله آسيبديديد

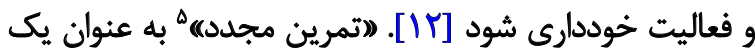

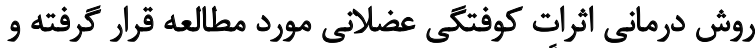

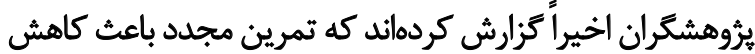

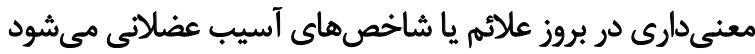

2. Eccentric resistance activities

3. Creatine Kinase (CK)

4. Lactate Dehydrogenase (LDH)

5. Re-training 
جدول (ـ مقايسه ميانكين و انحرافمعيار ويرُّكى هاى عمومى دو كروه

\begin{tabular}{|c|c|c|c|}
\hline \multirow{2}{*}{ سطح معنى دارى } & \multicolumn{2}{|c|}{ مياتكين \#اتحر اف معيار } & \multirow{2}{*}{ 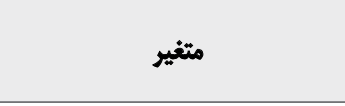 } \\
\hline & تمرين مجدد برون & تمرين برون & \\
\hline.$|P A|$ & rT/QYII/AT & $r \cdot / Y \pm V / \Delta$. & سن (سال) \\
\hline . & IVE/..tes/ar & $\mid V T / T \Delta \pm \Delta / M$ & قلد (سانتىمثر) \\
\hline Tחות & YD/RTIIY/A. & SQ/AYINTD & وزن (كيلوكرم) \\
\hline.$|\Delta V|$ & $m \in / . . \pm r / \ldots$ & $r Y / . . \pm V / T V$ & شاخص توده بدنى (كيلوكرم بر متر مربع) \\
\hline$\cdot / \pi \cdot \Delta$ & $1 r / . . \pm 1 / T q$ & $I T / \Lambda \Delta \pm \Delta / \pi f$ & جربى بلن (درصد) \\
\hline
\end{tabular}

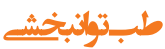

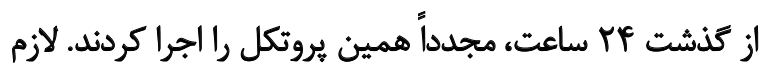

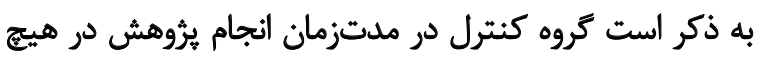
فعاليت ورزشى شركت نكردند.

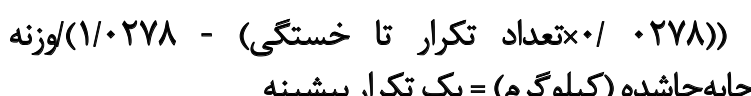

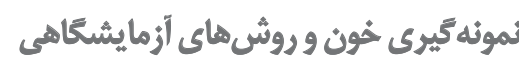

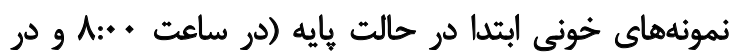

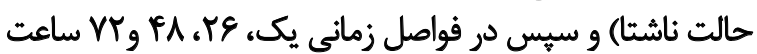

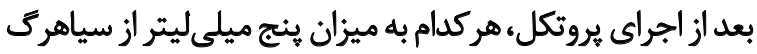

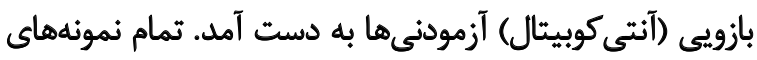

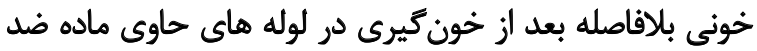

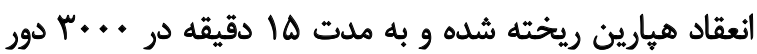

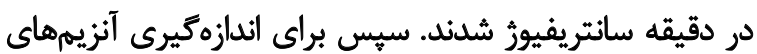

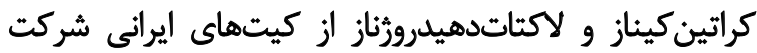

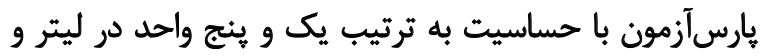

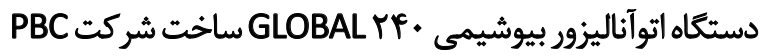

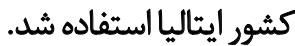

\section{سئجش الحساس درد عضلاني}

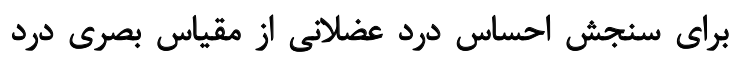

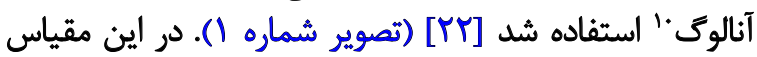

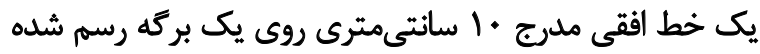

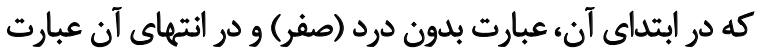

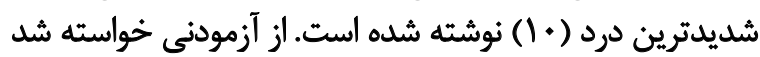

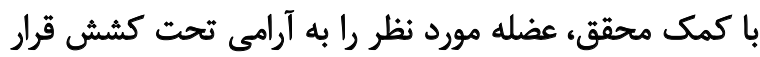

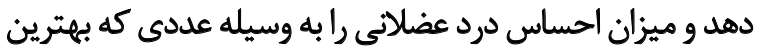

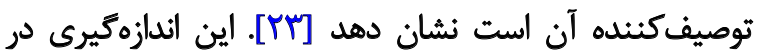

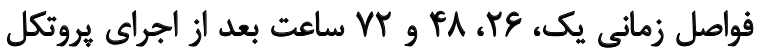

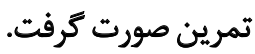

10. Visual Analog Scale (VAS)

\section{اندازٔميّيرى شاخص هاى أنثرويومتريك}

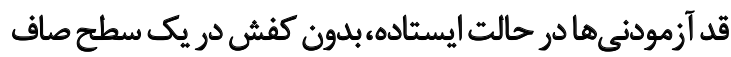

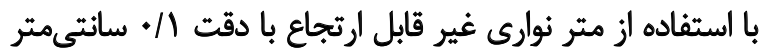

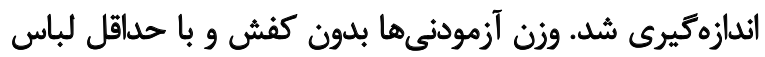

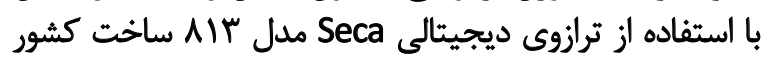

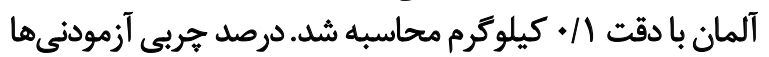

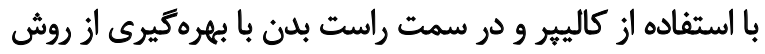

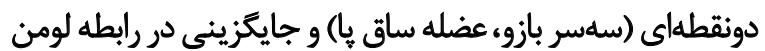

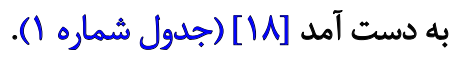

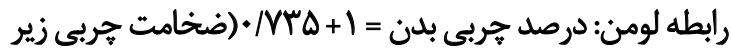

يوستى ساق ها + ضخامت هربى زير يوستى عضله سهسر بازو)

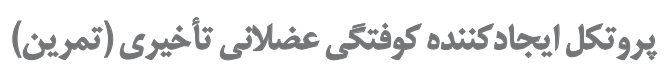

جلسه تمرين براى گروه آزمايش بدين صورت بود كه ابتدا

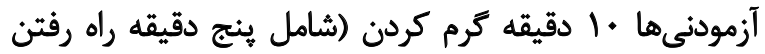

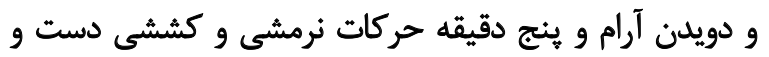

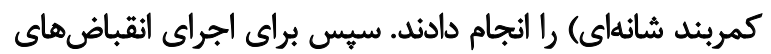

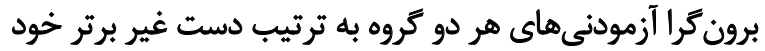

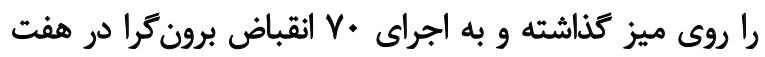

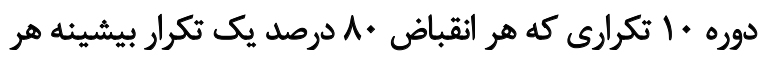

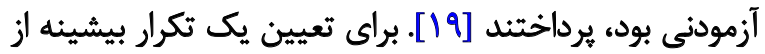

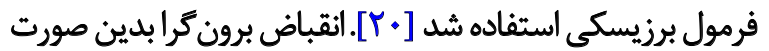

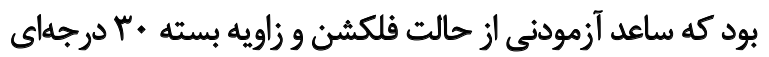

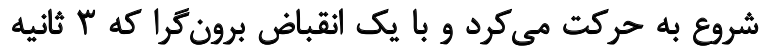

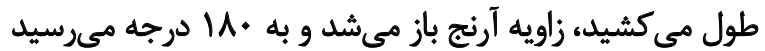

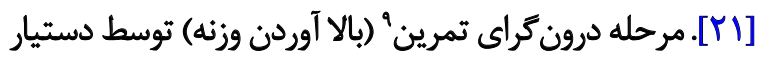

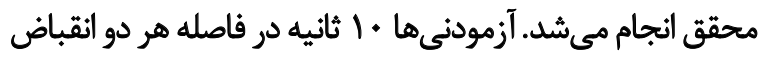

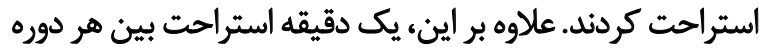

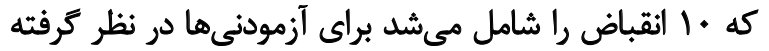

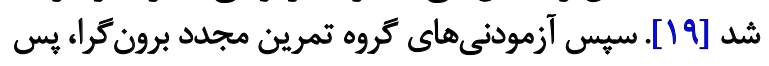

9. Concentric exercise 


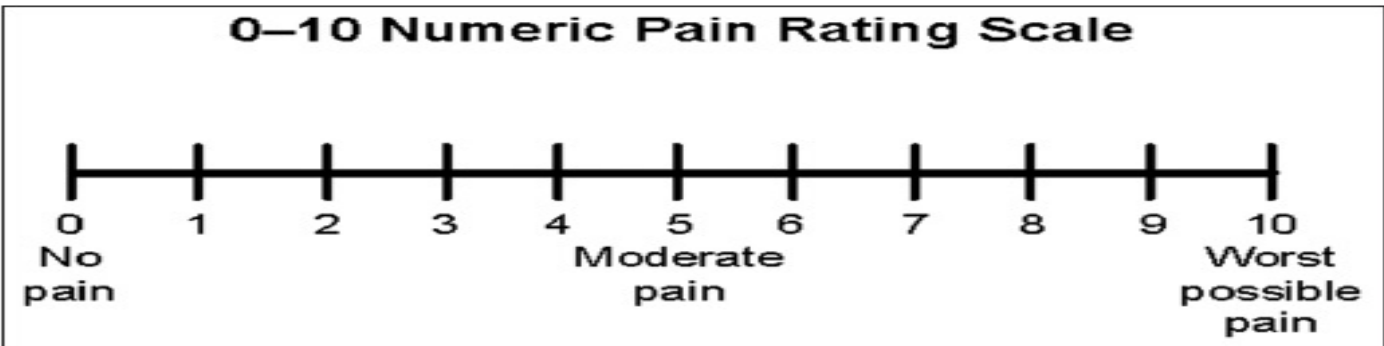

تصوير ا. مقياس بصرى درد آنالوى (VAS)

همجنين بر اساس نتايج بهدستآمده از آزمون تى مستقل،

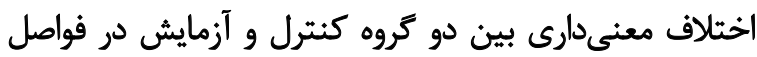

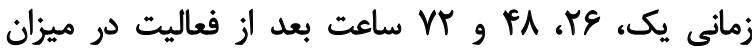

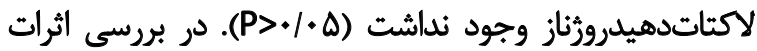

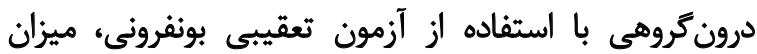

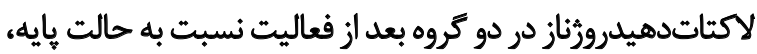

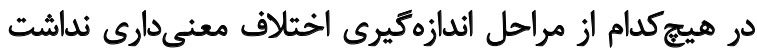

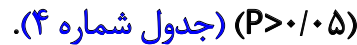

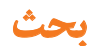

هدف از بروهش حاضر، بررسى ثأثير تمرين مجدد برونكرا

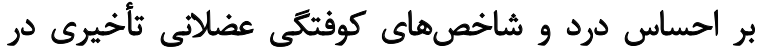

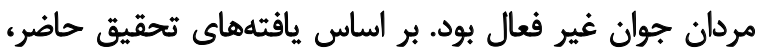

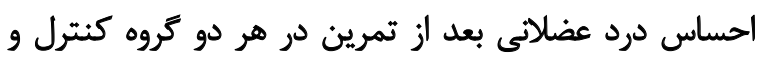

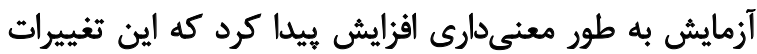

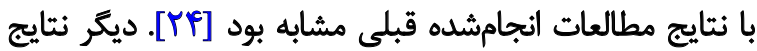

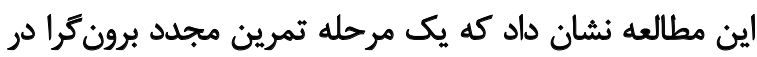

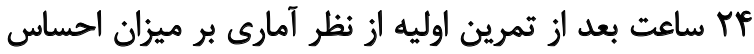

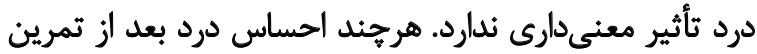

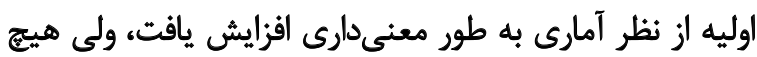

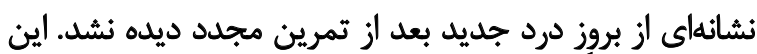

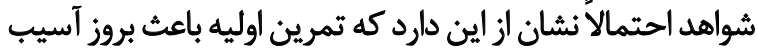

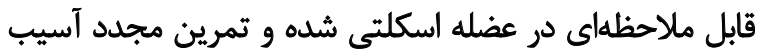

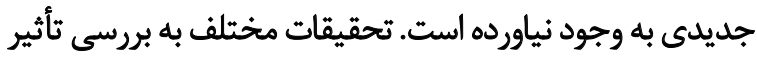

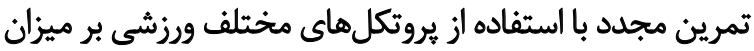

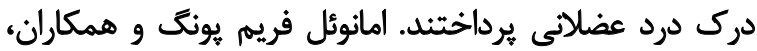

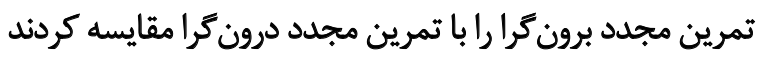

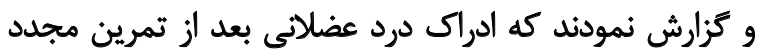

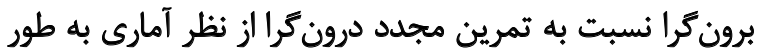

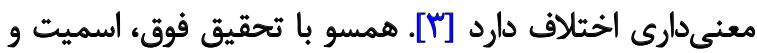

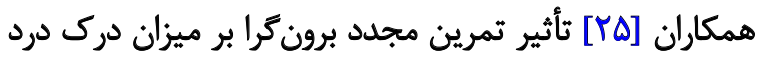

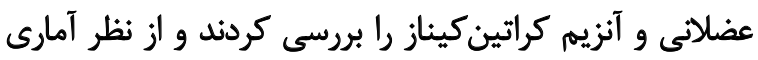

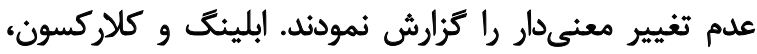

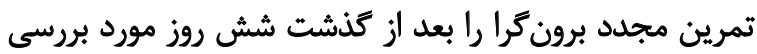

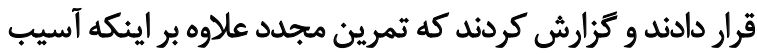

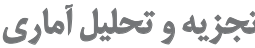

ابتدا از آزمون آمارى شاييرو ويلك جهت بررسى نرمال بودن إدمان

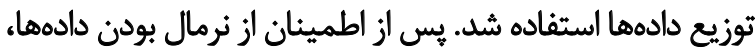

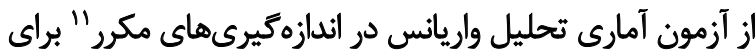

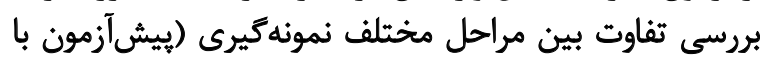

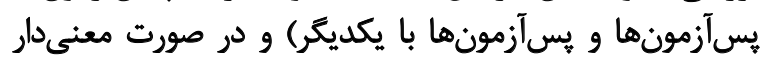

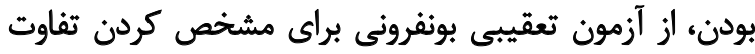

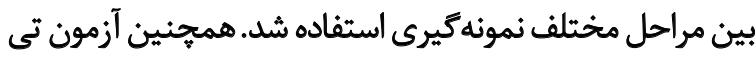

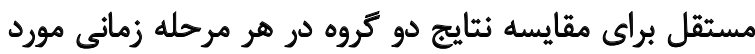

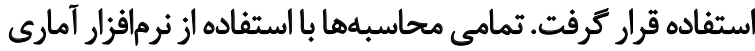

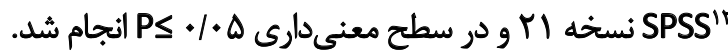

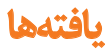

نتايج حاصل از آزمون تي مستقل نشان داد بين دو كروه كنترل

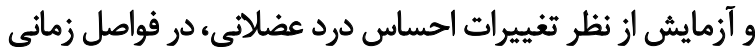

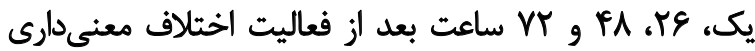

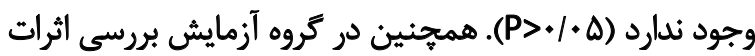

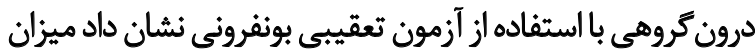

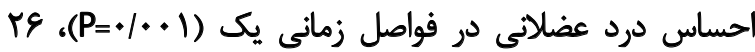

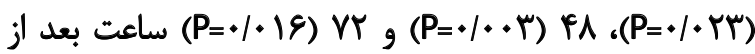

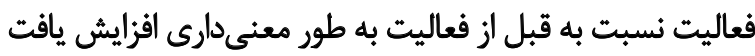
(جدول شماره r).

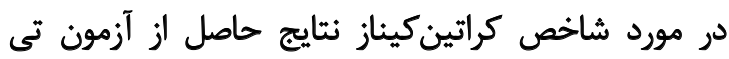

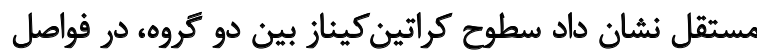

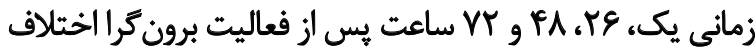

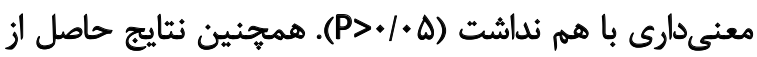

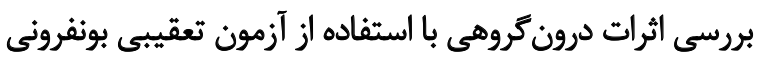

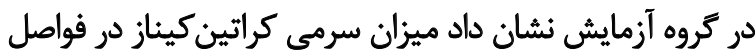

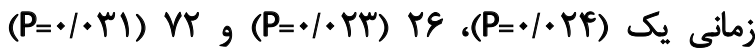

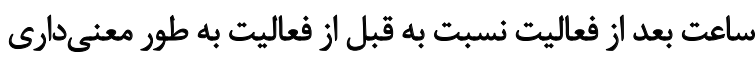

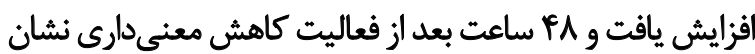

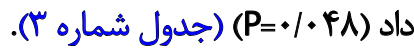

11. Analysis of variance in repeated measures

12. Statistical package for the social sciences 
جدول ب. مقايسه ميانكين و انحرافمعيار متغير وابسته احساس درد عضلانى قبل و بعد از تمرين، با آزمون تى مستقل و تى زوجى

\begin{tabular}{|c|c|c|c|}
\hline \multirow{2}{*}{ سطح معنى دارى" } & \multicolumn{2}{|c|}{ نمره درد (ميانكين ثانحرافمعيار) } & \multirow{2}{*}{ مرحله فعاليت بدنى } \\
\hline & كووه أزمايش & كروه كثترل & \\
\hline.$/ \Delta A \Delta$ & - & - & 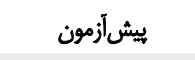 \\
\hline \multirow[b]{2}{*}{ - MAT } & $F / T V \pm 1 / .9$ & $r / \Delta \pm+/ \lambda r$ & يك ساعت بعد \\
\hline & .1 .1 & $.1 . .1$ & سطح معنى لارى "• \\
\hline \multirow[b]{2}{*}{ - MAT } & TVADV/ES & $r \pm . / 19$ & عا ساعت بعد \\
\hline & r & $+1 .+1$ & سطح معنى دارى" \\
\hline \multirow{2}{*}{.$/ 119$} & H/RTIV/A. & r/gs $\cdot|A|$ & مثا ساعت بعد \\
\hline & $\%$ & $.1 \cdot .1$ & سطح معنى لمارى" - \\
\hline \multirow{2}{*}{$. / F+\Lambda$} & $V / M T+/ Q t$ & $\cdot / N H \pm \cdot / V \Delta$ & ساعت بعل \\
\hline & .1 .18 & H.HT & سطح معنى لارى" " \\
\hline
\end{tabular}

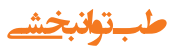

"سطح معنى دارى بين دو كروه، "سطح معنى دارى نسبت به حالت بايه (ييش آزمون).

آنزيم كراتينكيناز در افراد مختلف نسبت به تمرين برون كراى

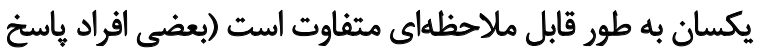

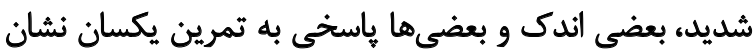

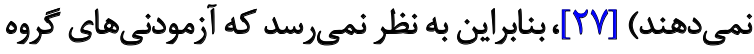

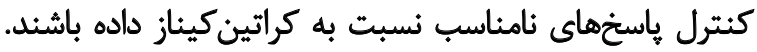

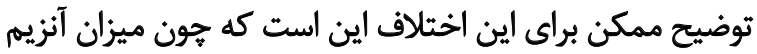

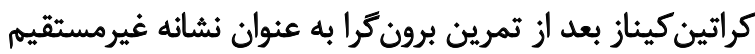

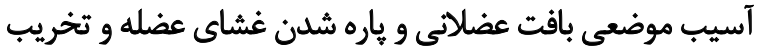

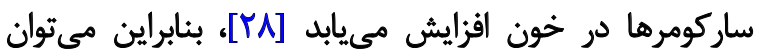

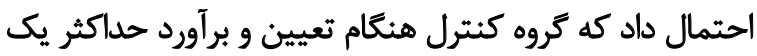

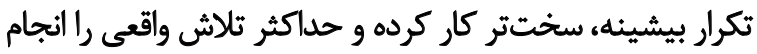

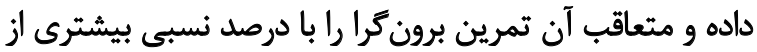

عضلانى را شديدتر نمى كند، باعث كاهش معنى دارى در ميزان

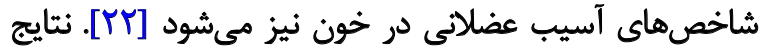

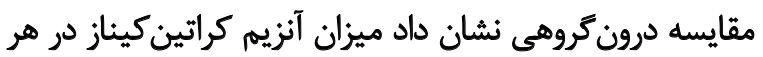

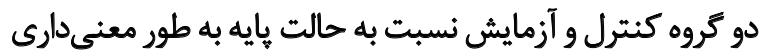

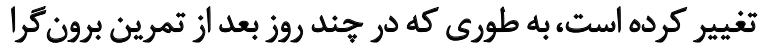

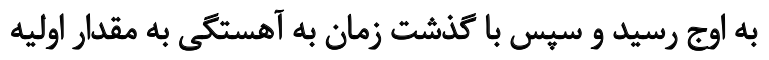

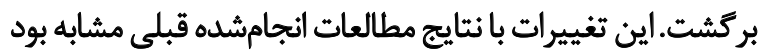

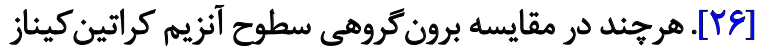

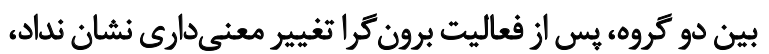

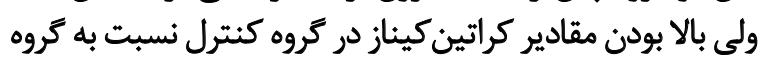

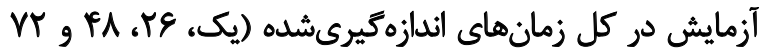
ساعت بعد) قابل توجه بود. بادر نظر كرفتن اينكه ميزان تغيير

جدول با. مقايسه ميانكين و انحرافمعيار متغير وابسته كراتين كيناز قبل و بعد از تمرين، با آزمون تى مستقل و تى زوجى

\begin{tabular}{|c|c|c|c|}
\hline \multirow{2}{*}{ سطح معنى دارى" } & \multicolumn{2}{|c|}{ ميزان كراتين كيناز برحسب واحد بينالملل بر ليتر (مياتعين +انحرافمعيار) } & \multirow{2}{*}{ 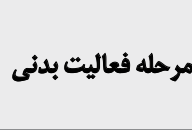 } \\
\hline & كروه آزمايش & كروه كثترل & \\
\hline.$/ 094$ & $109 \pm 9$. & $16 \% \pm 1.9$ & ي بيش آزمون \\
\hline \multirow{2}{*}{$.9 M T$} & $r|A \pm| r \mid$ & $M Y \pm I l P$ & يك ساعت بعد \\
\hline & ..$M E$ &.$/ . .4$ & سطح معنى دارى" \\
\hline \multirow{2}{*}{. /ATV } & rIVIIr. & rrrEIIf & عاعته بعل \\
\hline &.$/ 4 \pi$ &.$/ .48$ & سطح معنى لارى" " \\
\hline \multirow{2}{*}{ - IFAP } & $r \cdot \Delta \pm 1 \cdot r^{f}$ & riq土IIf & Aأراعت بعد \\
\hline & $*+\%$ & $1 . \mu$ & سطح معنى لارى" " \\
\hline \multirow{2}{*}{.$/ T V a$} & 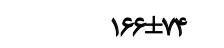 & $I W \pm A V$ & Thاعت بعد \\
\hline & $1 /+*+$ & $1 / *+$ & سطح معنى دارى" " \\
\hline
\end{tabular}


جدول f. مقايسه ميانكين و انحرافمعيار متغير وابسته لاكتاتدهيدروزناز قبل و بعد از تمرين با آزمون تى مستقل و تى زوجى

\begin{tabular}{|c|c|c|c|}
\hline \multirow{2}{*}{ سطح معنى داري" } & \multicolumn{2}{|c|}{ ميزان لاكتاتدهيدروزثاز برحسب واحد بينالملل بر ليتر (ميانكين土|نحرافمعيار) } & \multirow{2}{*}{ مرحله فعاليت بدنى } \\
\hline & كروه آزمايش & كروه كتترل & \\
\hline .1810 & $r q . \pm T$ & ragtry & ييش آزمون \\
\hline \multirow{2}{*}{ JV9ם } & $\varphi \| \pm$. & $M+ \pm T Q$ & يك ساعت بعد \\
\hline & - $/ A \Delta)^{\circ}$ & $1 / \ldots$ & سطح معنى دارى" \\
\hline \multirow{2}{*}{.$/ F \Delta F$} & fir $\pm \forall A$ & rratm & عابساعت بعد \\
\hline &.$/ 48$ & Vo.. & 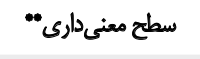 \\
\hline \multirow{2}{*}{$\cdot / A \cdot r^{\prime}$} & $f \cdot q \pm \lambda Y$ & $\forall 11 \pm \Delta q$ & مثاساعت بعد \\
\hline & 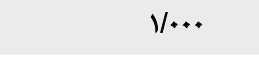 & 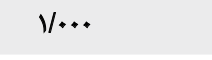 & 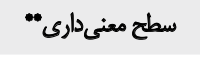 \\
\hline \multirow{2}{*}{.$/ 10 \Delta$} & rateq & PFA \pm Af & 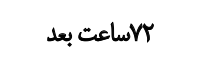 \\
\hline & $y / \ldots$ & .1119 & سطح معنى دارى" \\
\hline
\end{tabular}

\section{تتيجلكيرى}

بهطوركلي، نتايج اين مطالعه نشان داد اجراي تمرين مجدد

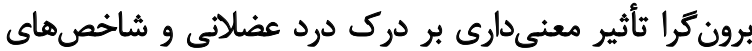

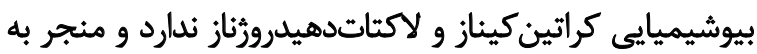

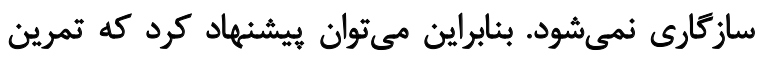

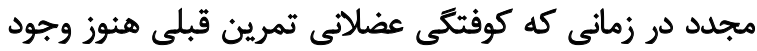

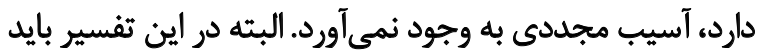

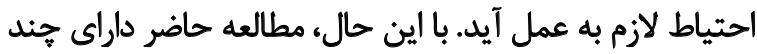

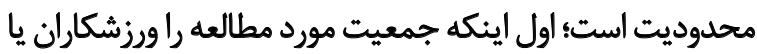

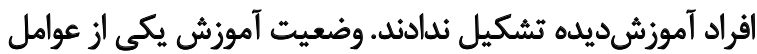

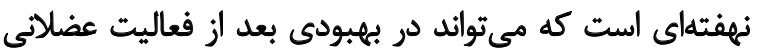

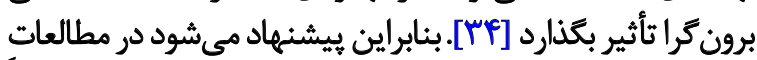

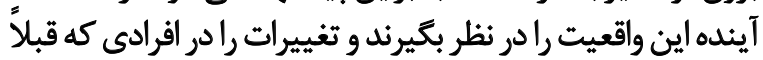

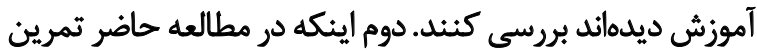

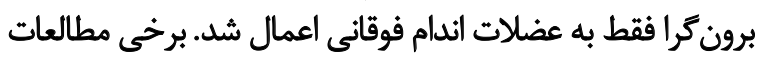

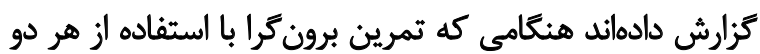

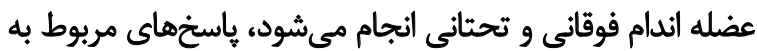

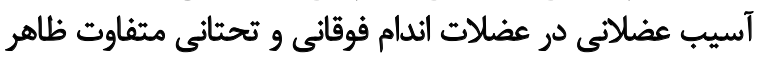

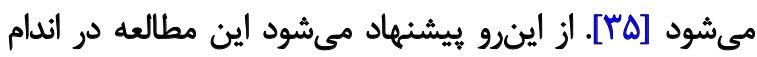

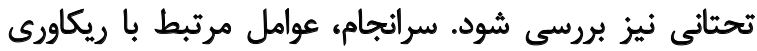

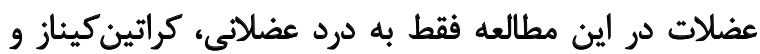

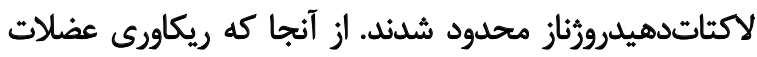

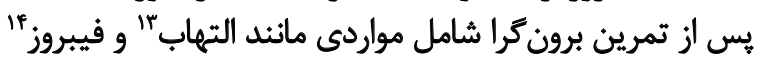

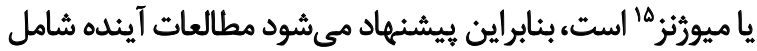

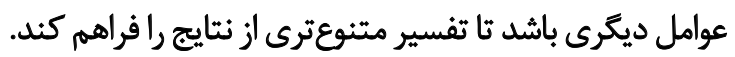

يك تكرار بيشينه نسبت به كروه آزمايش اجرا كرده است. بررسي

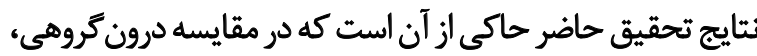

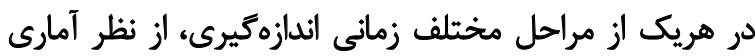

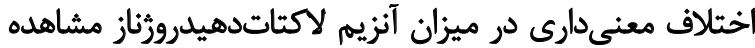

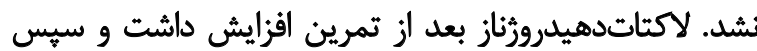

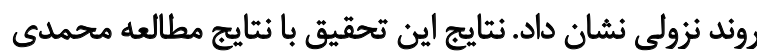

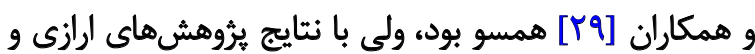

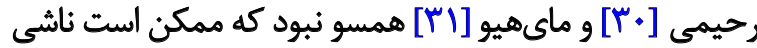

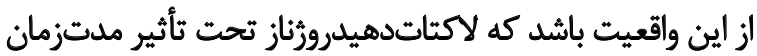

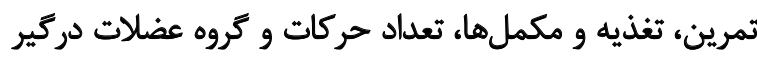

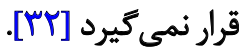

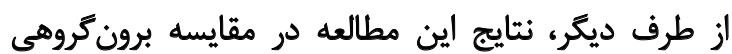

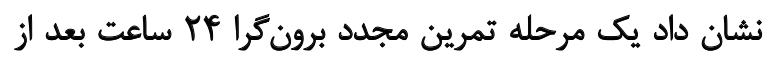

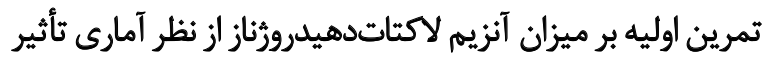

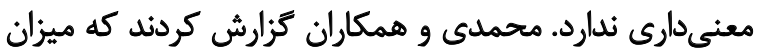

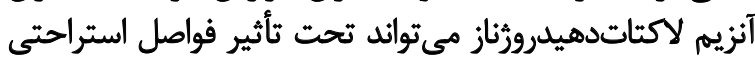

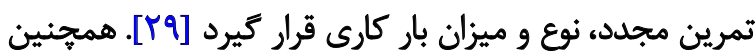

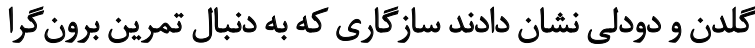

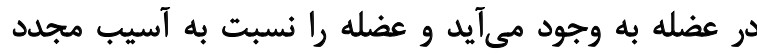

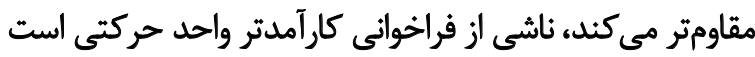

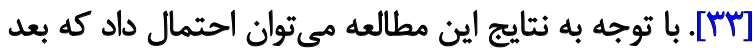

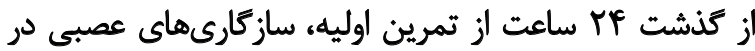

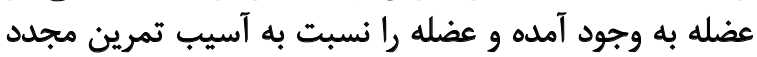
مقاومتر كرده است. بررسى تغييرات در فعاليت و فراخوانى واحد وديد

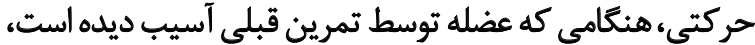
به مطالعات بيشترى نياز دارد. 


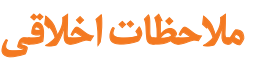

$$
\text { يميروى أز اصول اخلاق يثوهش }
$$

در اجراى يُروهش ملاحظات اخلاقي مطابق با دستورالعمل

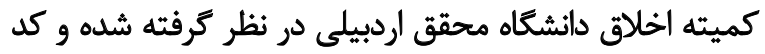

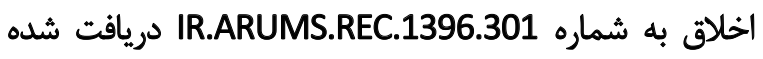

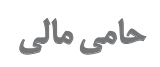

اين مقاله بركرفته از يايانتامه نويسنده اول در كروه فيزيولورثى

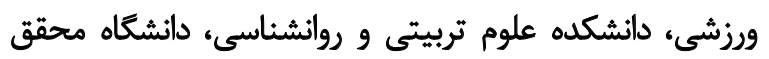

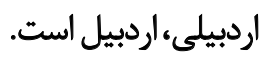

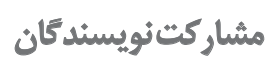

تمام نويسندكان در آمادهازى اين مقاله مشاركت يكسان

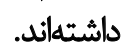

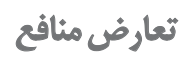

بنابر اظهار نويسندكان اين مقاله تعارض منافع ندارد.

$$
\text { تشكر و قدردانى }
$$

از دانشكاه محقق اردبيلى براي حمايتهاي همهجانبه تشكر و

قدرداني ميشود. 


\section{References}

[1] Timmons JA. Variability in training-induced skeletal muscle adaptation. Journal of Applied Physiology. 2011; 110(3):846-53. [DOI:10.1152/japplphysiol.00934.2010] [PMID] [PMCID]

[2] Hagen KB, Dagfinrud H, Moe RH, Østerås N, Kjeken I, Grotle $M$, et al. Exercise therapy for bone and muscle health: An overview of systematic reviews. BMC Medicine. 2012; 10:167. [DOI:10.1186/1741-7015-10-167] [PMID] [PMCID]

[3] Frimpong E, Ofori EK, Kaoje YS, Ababio E, Dzudzor B. Muscle Damage and repeated bout effect from high intensity non-eccentric exercises. Journal of Exercise Physiology Online. 2019; 22(5):126-39. https://www.asep.org/asep/asep/JEPonlineOCTOBER2019_Frimpong.pdf

[4] Kang MS, Kim J, Lee J. Effect of different muscle contraction interventions using an isokinetic dynamometer on muscle recovery following muscle injury. Journal of Exercise Rehabilitation. 2018; 14(6):1080-4. [DOI:10.12965/jer.1836440.220] [PMID] [PMCID]

[5] Nieman DC, Henson DA, McAnulty SR, McAnulty L, Swick NS, Utter AC, et al. Influence of vitamin $C$ supplementation on oxidative and immune changes after an ultramarathon. Journal of Applied Physiology. 2002; 92(5):1970-5. [DOI:10.1152/japplphysiol.00961.2001] [PMID]

[6] Barlas P, Craig JA, Robinson J, Walsh DM, Baxter GD, Allen JM. Managing delayed-onset muscle soreness: Lack of effect of selected oral systemic analgesics. Archives of Physical Medicine and Rehabilitation. 2000; 81(7):966-72. [DOI:10.1053/ apmr.2000.6277] [PMID]

[7] Howatson G, Van Someren KA. The prevention and treatment of exercise-induced muscle damage. Sports Medicine. 2008; 38(6):483-503. [DOI:10.2165/00007256-200838060-00004] [PMID]

[8] Cleary MA, Kimura IF, Sitler MR, Kendrick ZV. Temporal pattern of the repeated bout effect of eccentric exercise on delayedonset muscle soreness. Journal of Athletic Training. 2002; 37(1):32-6. [DOI:10.4320/164305.1293.7441]

[9] Hessel AL, Lindstedt SL, Nishikawa KC. Physiological mechanisms of eccentric contraction and its applications: A role for the giant titin protein. Frontiers in Physiology. 2017; 8:70. [DOI:10.3389/fphys.2017.00070] [PMID] [PMCID]

[10] Macaluso F, Isaacs AW, Myburgh KH. Preferential type II muscle fiber damage from plyometric exercise. Journal of Athletic Training. 2012; 47(4):414-20. [DOI:10.4085/1062-605047.4.13] [PMID] [PMCID]

[11] Margaritelis NV, Theodorou AA, Baltzopoulos V, Maganaris $\mathrm{CN}$, Paschalis V, Kyparos A, et al. Muscle damage and inflammation after eccentric exercise: Can the repeated bout effect be removed? Physiological Reports. 2015; 3(12):e12648. [DOI:10.14814/phy2.12648] [PMID] [PMCID]

[12] Armstrong R. Mechanisms of exercise-induced delayed onset muscular soreness: A brief review. Medicine and Science in Sports and Exercise. 1984; 16(6):529-38. [DOI:10.1249/00005768-198412000-00002]
[13] Mellinger S, Neurohr GA. Evidence based treatment options for common knee injuries in runners. Annals of Translational Medicine. 2019; 7(S7):S249. [DOI:10.21037/atm.2019.04.08] [PMID] [PMCID]

[14] Reimer III RC, Wikstrom EA. Functional fatigue of the hip and ankle musculature cause similar alterations in single leg stance postural control. Journal of Science and Medicine in Sport. 2010; 13(1):161-6. [DOI:10.1016/j.jsams.2009.01.001]

[15] Hyldahl RD, Chen TC, Nosaka K. Mechanisms and mediators of the skeletal muscle repeated bout effect. Exercise and Sport Sciences Reviews. 2017; 45(1):24-33. [DOI:10.1249/ JES.0000000000000095] [PMID]

[16] Donnelly AE, Clarkson PM, Maughan RJ. Exercise-induced muscle damage: Effects of light exercise on damaged muscle. European Journal of Applied Physiology and Occupational Physiology. 1992; 64(4):350-3. [DOI:10.1007/BF00636223] [PMID]

[17] Sorichter S, Koller A, Haid C, Wicke K, Judmaier W, Werne $P$, et al. Light concentric exercise and heavy eccentric muscle loading: Effects on CK, MRI and markers of inflammation. International Journal of Sports Medicine. 1995; 16(5):288-92. [DOI:10.1055/s-2007-973007] [PMID]

[18] Lohman TG. Advances in body composition assessment. Cham paign: Human Kinetics Publishers; 1992. https://books.google. com/books/about/Advances_in_Body_Composition_Assessment.html?id=euuUMQEACAAJ\&source=kp_book_description

[19] Nejatmand N, Ramezani A, Barati AH. [Effect of Consumption short-term CoQ10 supplementation on markers of delayed onset muscle soreness (Persian)]. Razi Journal of Medical Sciences. 2014; 21(119):77-85. http://rjms.iums.ac.ir/article1-3105-en.html

[20] Brzycki M. Strength testing-predicting a one-rep max from repsto-fatigue. Journal of Physical Education, Recreation \& Dance. 1993; 64(1):88-90. [DOI:10.1080/07303084.1993.10606684]

[21] Latella C, Goodwill AM, Muthalib M, Hendy AM, Major B, Nosaka $K$, et al. Effects of eccentric versus concentric contractions of the biceps brachii on intracortical inhibition and facilitation. Scandinavian Journal of Medicine \& Science in Sports. 2019; 29(3):369-79. [DOI:10.1111/sms.13334] [PMID]

[22] Ebbeling CB, Clarkson PM. Muscle adaptation prior to recovery following eccentric exercise. European Journal of Applied Physiology and Occupational Physiology. 1990; 60(1):26-31. [DOI:10.1007/BF00572181] [PMID]

[23] Miles M, Clarkson P. Exercise-induced muscle pain, soreness, and cramps. The Journal of Sports Medicine and Physical Fitness. 1994; 34(3):203-16. [doi:10.1038/bjv.7830383]

[24] Hody S, Croisier J-L, Bury T, Rogister B, Leprince P. Eccentric muscle contractions: Risks and benefits. Frontiers in Physiology. 2019; 10:536. [DOI:10.3389/fphys.2019.00536] [PMID] [PMCID]

[25] Smith L, Fulmer M, Holbert D, McCammon MR, Houmard $J A$, Frazer $D$, et al. The impact of a repeated bout of eccentric exercise on muscular strength, muscle soreness and creatine kinase. British Journal of Sports Medicine. 1994; 28(4):267-71. [DOI:10.1136/bjsm.28.4.267] [PMID] [PMCID] 
[26] Kim HT. Effect of hydroxytyrosol supplementation on muscle damage in healthy human following an acute bout of exercise [MSc. thesis]. Texas: The University of Texas at Austin; 2013. https://repositories.lib.utexas.edu/ handle/2152/22280?show=full

[27] Newham D, Mills K, Quigley B, Edwards R. Pain and fatigue after concentric and eccentric muscle contractions. Clinical Science. 1983; 64(1):55-62. [DOI:10.1042/cs0640055] [PMID]

[28] Ebbeling CB, Clarkson PM. Exercise-induced muscle damage and adaptation. Sports Medicine. 1989; 7(4):207-34. [DOI:10.2165/00007256-198907040-00001] [PMID]

[29] Mohammadi H, Afzalpour ME, levary SHA. Response of creatine kinase and lactate dehydrogenase enzymes to rest interval between sets and set-repetition configuration during bouts of eccentric exercise. Interventional Medicine and Applied Science. 2018; 10(2):83-6. [DOI:10.1556/1646.10.2018.09] [PMID] [PMCID]

[30] Arazi H, Rahimi R. The effect of different rest intervals between multiple bench press bouts. South African Journal for Research in Sport, Physical Education and Recreation. 2011; 33(1):1-8. [DOI:10.4314/sajrs.v33i1.65480]

[31] Mayhew DL, Thyfault JP, Koch AJ. Rest-interval length affects leukocyte levels during heavy resistance exercise. Journal of Strength and Conditioning Research. 2005; 19(1):16-22. [DOI:10.1519/R-14113.1] [PMID]

[32] Hosseini E, Daneshjoo A, Sahebozamani M, Behm D. The effects of fatigue on knee kinematics during unanticipated change of direction in adolescent girl athletes: A comparison between dominant and non-dominant legs. Sports Biomechanics. 2021:1-10. [DOI:10.1080/14763141.2021.1925732]

[33] Golden CL, Dudley GA. Strength after bouts of eccentric or concentric actions. Medicine and Science in Sports and Exercise. 1992; 24(8):926-33. [DOI:10.1249/00005768199208000-00015]

[34] Kim J, Lee J. The relationship of creatine kinase variability with body composition and muscle damage markers following eccentric muscle contractions. Journal of Exercise $\mathrm{Nu}$ trition \& Biochemistry. 2015; 19(2):123-9. [DOI:10.5717/ jenb.2015.15061910] [PMID] [PMCID]

[35] Saka T, Akova B, Yazici Z, Sekir U, Gür H, Ozarda Y. Difference in the magnitude of muscle damage between elbow flexors and knee extensors eccentric exercises. Journal of Sports Science \& Medicine. 2009; 8(1):107-15. [DOI:10.1373/jmed.24150563] 